\title{
On Quasi-Optimum Detection of Nonlinearly Distorted OFDM Signals
}

\author{
João Guerreiro $^{1,2}$, Rui Dinis ${ }^{1,2}$, and Paulo Montezuma ${ }^{1,3}$ \\ ${ }^{1}$ DEE, FCT, Universidade Nova de Lisboa, Monte de Caparica, Portugal \\ ${ }^{2}$ IT, Instituto de Telecomunicações, Lisboa, Portugal \\ ${ }^{3}$ UNINOVA, Monte de Caparica, Portugal
}

\begin{abstract}
In this paper we considered OFDM schemes that somehow have a nonlinear operation on their transmission chain. Contrary to the conventional OFDM implementations, we consider the distortion term that come from that operation as something useful that has information on the transmitted signals. To efficiently harvest this information, we develop an optimum-based receiver that presents good performance improvements without introduce very high complexities. The performance of this sub-optimal receiver is investigated under different types of channel and considering receive diversity. ${ }^{1}$
\end{abstract}

Keywords: OFDM signals, nonlinear distortion effects, optimum receiver, Euclidean distance.

\section{Introduction}

OFDM (Orthogonal Frequency Division Multiplexing) [1] schemes support the physical layer of several wireless communications standards such as DVB [2], LTE [3] and WiMAX [4]. The reasons behind their popularity reside mainly on their facility to cope with frequency-selective channels without the need for complex equalization processes and their simplicity of implementation. However, OFDM signals have a critical issue: they present a very high Peak-to-Average Power Ratio (PAPR). If nothing is done, this will cause severe amplification difficulties and strong nonlinear distortion effects at the transmitter output. As the use of an high Input Back-Off (IBO) is not a good solution due to the inherent energy inefficiency, several techniques that aim to reduce the envelope fluctuations of OFDM signals have been proposed in the literature [5]. Between them, the simplest one involves the use of an envelope clipper followed by a frequency-domain filter (FDF), which allows an efficient PAPR reduction without introduce intolerable complexities and compromise the spectral efficiency [6]. However, although the clipped signals are linearly amplified with ease, they will also present nonlinear distortion effects since they are generated through a nonlinear operation.

\footnotetext{
${ }^{1}$ This work was supported by the FCT/MEC projects CTS PEst-OE/EEI/UI0066/ 2011, IT PEst-OE/EEI/LA0008/ 2013， OPPORTUNISTIC-CR PTDC/EEA-TEL/115981/ 2009, ADCOD PTDC/EEA-TEL/099973/2008, ADIN PTDC/EEI-TEL/2990/2012 and Femtocells PTDC/EEA-TEL/120666/2010 as well as by grant SFRH/BD/90997/2012.
} 
It is well known that the samples of an OFDM signal are well modeled by a Gaussian distribution, specially when a high number of subcarriers is considered. This approximation allows the use of the Bussgang theorem [7] that states that a nonlinearly distorted OFDM signal can be divided into two uncorrelated components: an useful term that is proportional to the transmitted signal and a second one that represents the nonlinear distortion. Considered as noise in the typical OFDM implementations, the distortion term is seen as something that degrades the performance. To avoid this, there are receivers that try to estimate and cancel this distortion [8], but their usefulness are very limited specially at high Signal-to-Noise Ratios (SNRs). In this work we considered not only to accept the nonlinear distortion but also to use it as something that can effectively improve the performance of the nonlinear OFDM schemes. In fact, the distortion term has useful information on the transmitted signals that can be used to improve the performance [9]. The best way to use this information is to consider an optimum receiver that makes an estimate of the transmitted signal based on the Euclidean Distance, which is evaluated using the two terms of the Bussgang Theorem. The research question can be stated as: can the nonlinear OFDM schemes perform better than the linear ones with the use of optimum receivers? To answer this question, we adopted a research approach based on the study of the relation between the Euclidean distance and the average bit energy with and without nonlinear distortion effects, which allows to conclude about the expected Bit Error Rate (BER). Surprisingly, it was verified that the nonlinear OFDM schemes can have better performances than the linear ones. As the complexity of the optimum receiver is very high even considering a small number of subcarriers, we propose a reduced-complexity receiver that in spite of not performing a full optimum detection can also achieve excellent performances.

The paper is divided in the following sections: In Sec. 2 we present the relationship between this work and the collective awareness systems (CAS). Sec. 3 concerns about the communication scenario that is used as the basis of this work as well as the principal characteristics of nonlinearly distorted OFDM signals modeled with a Gaussian approximation. In Sec. 4 the optimum detection is introduced and the potential performance of the optimum receiver is presented. Sec. 5 presents a sub-optimal received and shows its performance results under different scenarios. Finally, Sec. 6 concludes this paper and presents some directions of future work.

\section{Relationship to Collective Awareness Systems}

CAS are today one of the biggest technological challenges in the digital world. The idea of everyone to communicate and use collective knowledge to decide in real-time presupposes solid communication mechanisms that can provide a good Quality of Service (QoS) without high energy consumption. Behind the real-time decisions, it is easily to see that wireless communications have a very important role. This work aims to present improvements in the quality of wireless communications that use OFDM modulations, which is the case of the most part of the standards, by reducing the energy consumption that is need to achieve a specific error rate and hence improving the energy efficiency of the equipments that compose the collective awareness systems. 


\section{Nonlinear OFDM Schemes}

In this section we characterize the considered nonlinear OFDM scenario by describing the signals along the transmission chain depicted in Fig.2. We represent the baseband data signal with the vector $\mathbf{S}=\left[\begin{array}{llll}S_{0} S_{1} & \ldots & S_{N M-1}\end{array}\right]^{T} \in \mathbb{C}^{N M}$, where $N$ is the number of subcarriers considered in each OFDM block and $M$ is the oversampling factor. Each complex data symbol $S_{k}$ is selected from an $\mathcal{M}$-order QAM constellation (e.g. 4-QAM). The time-domain version $\mathbf{S}$ is obtained through an Inverse Discrete Fourier Transform (IDFT), i.e., $\mathbf{s}=\mathbf{F}^{-1} \mathbf{S}=\left[\begin{array}{llll}s_{0} & s_{1} & \ldots & s_{N M-1}\end{array}\right]^{T} \in \mathbb{C}^{N M}$, where $\mathbf{F}$ represents the Discrete Fourier Transform (DFT) matrix with the $\left(n, n^{\prime}\right)$ element defined by

$$
F_{n, n \prime}=\frac{1}{\sqrt{N M}} \exp \left(-\frac{j 2 \pi n n^{\prime}}{N M}\right)
$$

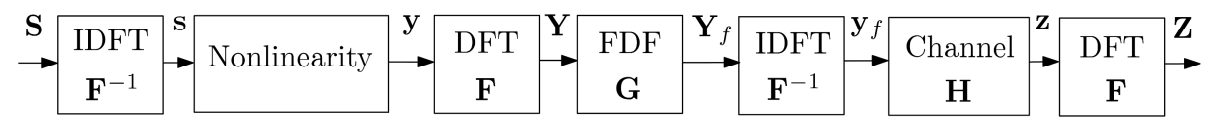

Fig. 1. Basic nonlinear OFDM scheme

If high values of $N$ are considered (let's say $N \geq 32$ ), the imaginary and the real parts of the time-domain samples represented by $\mathbf{s}$ can be modeled by a Gaussian distribution. Representing $\bar{S}_{I}$ and $\bar{S}_{Q}$ as the considered Gaussian random variables, we can write

$$
p_{\bar{s}_{I}}\left(s_{I}\right)=\frac{1}{\sigma \sqrt{2 \pi}} \exp \left(-\frac{\left(s_{I}-\mu\right)^{2}}{2 \sigma^{2}}\right)
$$

and $p\left(s_{I}\right)=p\left(s_{Q}\right)$. The absolute value of $\mathbf{s}$ is represented by $\mathbf{R}=|\mathbf{s}|=$ $\left[\begin{array}{llll}R_{0} & R_{1} & \ldots & R_{N M-1}\end{array}\right]^{T} \in \mathbb{R}^{N M}$ and modeled by $\bar{R}$, which is a Rayleigh-distributed random variable with its PDF given by

$$
p_{\bar{R}}(R)=\frac{R}{\sigma^{2}} \exp \left(-\frac{R^{2}}{2 \sigma^{2}}\right) u(R)
$$

with $u(\bar{R})$ denoting the unitary step function and $\sigma^{2}=\operatorname{var}\left(\bar{S}_{I}\right)=\operatorname{var}\left(\bar{S}_{Q}\right)$, denoting the variance of the real and imaginary parts that is assumed to be equal. Under the Gaussian approximation, the Bussgang theorem [7] states that the nonlinearly distorted signal represented by $\mathbf{y}=f(|\mathbf{s}|)=f(\mathbf{R})=\left[\begin{array}{llll}y_{0} & y_{1} & \ldots & y_{N M-1}\end{array}\right]^{T} \in \mathbb{C}^{N M}$ can be decomposed in two terms, i.e.,

$$
\mathbf{y}=\alpha \mathbf{s}+\mathbf{d},
$$

where $\mathbf{s}$ is the time-domain version of the modulated signal, $\mathbf{d}=\left[\begin{array}{llll}d_{0} & d_{1} & \ldots & d_{N M-1}\end{array}\right]^{T} \in \mathbb{C}^{N M}$ is the distortion term and $\alpha$ is a scaling factor given by 


$$
\alpha=\frac{\mathbb{E}\left[R_{n} f\left(R_{n}\right)\right]}{\mathbb{E}\left[R_{n}^{2}\right]} .
$$

The nonlinearity considered in our transmission chain is assumed to be memoryless. These nonlinearities can be modeled by the Saleh model [10] that characterizes the output of a nonlinear device using its AM-AM and AM-PM conversion functions, both receiving the absolute value of the signal as input (and represented by $A(\cdot)$ and $\Theta(\cdot)$, respectively). Using this model, we can also write the $n$-th output sample as

$$
f\left(R_{n}\right)=A\left(R_{n}\right) \exp \left(j\left(\arg \left(s_{n}\right)+\Theta\left(R_{n}\right)\right),\right.
$$

with $\arg \left(s_{n}\right)$ being the original phase of the $n$-th time-domain sample. The considered memoryless nonlinearity is an ideal envelope clipper with normalized clipping level $s_{M} / \sigma$,

$$
f\left(R_{n}\right)= \begin{cases}R_{n}, & R_{n} \leq s_{M} / \sigma \\ s_{M} \exp \left(j \arg \left(s_{n}\right)\right), & R_{n}>s_{M} / \sigma\end{cases}
$$

Note that in this case $f\left(R_{n}\right)=A\left(R_{n}\right) \exp \left(j \arg \left(s_{n}\right)\right)$, since we don't have phase distortion. The use of a normalized clipping level is related to the random nature of our signals. A low $s_{M} / \sigma$ means that the random samples of the signal will enter in the nonlinear region very often. Making use of the DFT definition of (1), we can express the frequency version of (4) by

$$
\mathbf{Y}=\mathbf{F} \mathbf{y}=\alpha \mathbf{S}+\mathbf{D},
$$

where $\mathbf{D}=\left[\begin{array}{llll}D_{0} & D_{1} & \ldots & D_{N M-1}\end{array}\right]^{T} \in \mathbb{C}^{N M}$ is the frequency-domain version of the distortion term that can be modeled by $\bar{D}$, which is a Gaussian random variable with zero mean as shown in [6]. In order to maintain a good spectral efficiency and the bandwidth of the original transmitted signal, a frequency-domain filter (FDF) must be used to eliminate the out-of-band radiation inherent to the nonlinear operation. In our signal processing scheme, the filtering operation is represented as a

multiplication by the diagonal matrix $\mathbf{G}$, that is defined as

$$
\mathbf{G}=\operatorname{diag}([\underbrace{0 \ldots 0}_{(M-1) N / 2} \underbrace{1 \ldots \ldots 1}_{N} \underbrace{0 \ldots 0}_{(M-1) N / 2}]) .
$$

The filtered signal is given by

$$
\mathbf{Y}_{f}=\mathbf{G Y} \text {. }
$$

At the reception side (after the channel effect), we have the time-domain block $\mathbf{z}=\left[\begin{array}{lllll}z_{0} & z_{1} & \ldots & Z_{N M-1}\end{array}\right]^{T} \in \mathbb{C}^{N M}$ with the corresponding frequency-domain block $\mathbf{Z}=\left[\begin{array}{lllll}Z_{0} & Z_{1} & \ldots & Z_{N M-1}\end{array}\right]^{T} \in \mathbb{C}^{N M}$ defined by

$$
\mathbf{Z}=\mathbf{H Y}_{\mathbf{f}}+\mathbf{N}=\mathbf{H G F f}\left(\mathbf{F}^{-1} \mathbf{S}\right)+\mathbf{N}=\alpha \mathbf{H G S}+\mathbf{H G D}+\mathbf{N},
$$


where $\mathbf{N}=\left[\begin{array}{llll}N_{0} & N_{1} & \ldots & N_{N M-1}\end{array}\right]^{T} \in \mathbb{C}^{N M}$ represents the noise components and

$$
\mathbf{H}=\operatorname{diag}\left(\left[\begin{array}{llll}
H_{0} & H_{1} & \ldots & H_{N M-1}
\end{array}\right]^{T}\right),
$$

represents the channel frequency responses.

\section{Optimum Detection and Its Potential Performance}

The presence of nonlinear distortion effects on the transmitted signals is typically seen as something undesirable. However, the optimum detection can take advantage of the distortion introduced by the nonlinearity for performance improvements. To understand this, let us first recall that the optimum receiver estimate is obtained by minimizing the least square error (LSE) between the received signal $\mathbf{Z}$ and a possible transmitted signal, HY $=\mathbf{H F} f\left(\mathbf{F}^{-1} \mathbf{S}\right)$. To evaluate the LSE between two vectors we can compute the squared Euclidean norm between them, which makes possible to write that the optimum estimate is given by

$$
\widehat{\mathbf{S}}=\min _{\mathbf{S}}|| \mathbf{Z}-\mathbf{H F} f\left(\mathbf{F}^{-1} \mathbf{S}\right)||^{2},
$$

which, in fact, is the minimum Euclidean distance between $\mathbf{H Y}=\mathbf{H F} f\left(\mathbf{F}^{-1} \mathbf{S}\right)$ and $\mathbf{Z}$. Note that to choose the best sequence, all the possible transmitted signals must be evaluated. Here the term "evaluated" means that the possible transmitted sequence must be submitted to the same signal processing chain of the received signal. Let us also recall that what strongly conditions the BER of a communication system is the ratio between the average bit energy, $E_{b}$, and the minimum squared Euclidean Distance between the transmitted signals, $\mathcal{D}^{2}$. In the presence of nonlinear distortion effects, this ratio is higher and, consequently, we can have potential performance improvements. To verify this, let us consider two nonlinearly distorted OFDM signals that differ in one symbol, $\mathbf{Y}^{(2)}=\mathbf{F} f\left(\mathbf{F}^{-1} \mathbf{S}^{(2)}\right)$ and $\mathbf{Y}^{(1)}=\mathbf{F} f\left(\mathbf{F}^{-1} \mathbf{S}^{(1)}\right)$ associated to the modulated data signals $\mathbf{S}^{(2)}$ and $\mathbf{S}^{(1)}$, respectively. The Euclidean distance between $\mathbf{S}^{(2)}$ and $\mathbf{S}^{(1)}$ is

$$
\mathcal{D}^{2}=|| \mathbf{S}^{(2)}-\mathbf{S}^{(1)}||^{2}=\sum_{k=1}^{N}\left|S_{k}^{(2)}-S_{k}^{(1)}\right|^{2}
$$

With normalized QPSK constellations (i.e. $S_{k}= \pm 1 \pm j$ ), we have $\mathcal{D}^{2}=4 E_{b}$, with

$$
E_{b}=\frac{\|\mathbf{Y}\|^{2}}{\log _{2}(\mathcal{M}) N_{\mathcal{M}=4}}=1
$$

Considering that the signals are nonlinearly distorted and using (8), the Euclidean Distance is given by

$$
\mathcal{D}_{N L}^{2}=|| \mathbf{Y}^{(2)}-\mathbf{Y}^{(1)} \|^{2}=\sum_{k=1}^{M N}\left|\alpha\left(S_{k}^{(2)}-S_{k}^{(1)}\right)+D_{k}^{(2)}-D_{k}^{(1)}\right|^{2},
$$


and typically we have $\mathcal{D}_{N L}^{2}>4 E_{b}$. In these conditions we can define an asymptotic gain given by

$$
\mathcal{G}=10 \log _{10}\left(\frac{\mathcal{D}_{N L}^{2}}{\mathcal{D}^{2}}\right)
$$

In Fig. 1 is shown the probability density function (PDF) of (17) without considering an FDF (i.e., the diagonal of $\mathbf{G}$ is zeros) and considering OFDM signals with $N=64$ useful subcarriers distorted by an ideal envelope clipper with normalized clipping level $s_{M} / \sigma$. It is assumed an ideal AWGN channel (i.e., $\left|H_{k}\right|=1 \forall k$ ) and $\mu$ different symbols between $\mathbf{S}^{(2)}$ and $\mathbf{S}^{(1)}$.

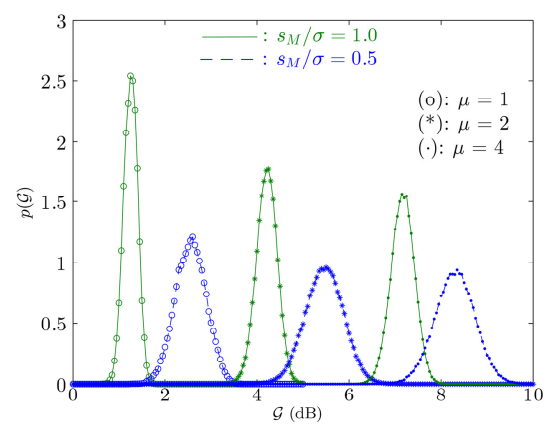

Fig. 2. Distribution of the asymptotic gain for different values of $s_{M} / \sigma$ and $\mu$

From Fig. 2, it is clear that gain is almost higher than one. Moreover, the stronger the nonlinear distortion effects the higher is the magnitude of the gain. For instance, with $\mu=1$ and $s_{M} / \sigma=1.0$, we have an asymptotic gain around $1.4 \mathrm{~dB}$, but the gain can even reach $2.7 \mathrm{~dB}$ for $s_{M} / \sigma=0.5$ and the same value of $\mu$. From the figure it is also clear another important aspect: the average value of the gain is proportional to $\mu$, i.e., $\mathbb{E}[\mathcal{G}]_{\mu=a}=a \mathbb{E}[\mathcal{G}]_{\mu=1}$, which unveils that even in a coded scenario where $\mu$ depends on the free distance of the code and is typically higher than one, we will also have potential performance improvements. To verify the asymptotic gains associated to the optimum detection in nonlinear OFDM schemes, we obtain the BER using the following approximation (that is valid considering that the minimum Euclidean distance is dominated by sequences that differ in only $\mu=1$ symbols)

$$
P_{b}=\sum_{i} P\left(\mathcal{G}=\mathcal{G}_{i}\right) Q\left(\sqrt{\mathcal{G}_{i} \frac{2 E_{b}}{N_{0}}}\right)
$$

with $\mathcal{G}_{i}$ being the value that the gain can takes (measured in linear units) and $P\left(\mathcal{G}=\mathcal{G}_{i}\right)$ is the probability of the gain $\mathcal{G}$ be equal to $\mathcal{G}_{i}$. In Fig.2 it is shown the BER obtained using (18) and considering OFDM signals with $N=64$ subcarriers and different normalized clipping levels $s_{M} / \sigma$. 


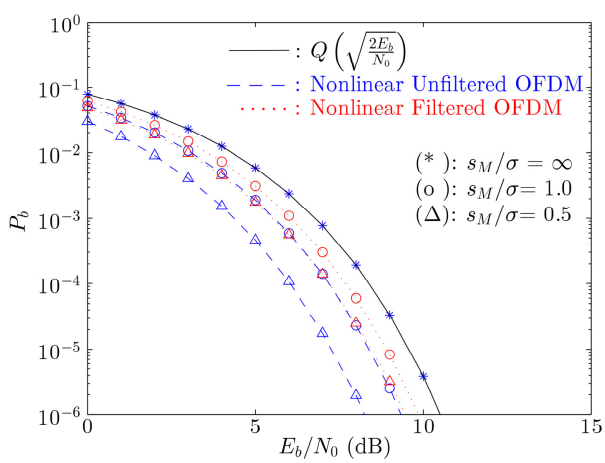

Fig. 3. Approximate BER for different values of $s_{M} / \sigma$ with and without FDF

From the Fig. 3 it is clear that in the case of linear OFDM, $P(\mathcal{G}=1)=1$ which means that $\mathcal{D}_{N L}^{2}=\mathcal{D}^{2}=4 E_{b}$ and the approximate BER of (18) is equal to the theoretical linear OFDM BER given by $P_{b}=Q\left(\sqrt{\frac{2 E_{b}}{N_{0}}}\right)$. As is expected from the gains distribution, when the nonlinear distortion effects are stronger the potential improvements in the BER are higher too. At $P_{b}=10^{-3}$ and $s_{M} / \sigma=0.5$ the asymptotic gain relative to the linear case is around $2.6 \mathrm{~dB}$. When we consider a FDF to remove the out-of-band energy inherent to the nonlinear operation, the gains are lower, which is an expected result since less subcarriers are considered in the computation of the Euclidean distance, i.e., $\mathcal{D}_{N L}^{2}$ is computed only on the $N$ in-band subcarriers. However, even considering filtered sequences, the asymptotic gain can reach a value around $1.3 \mathrm{~dB}$.

\section{Sub-optimal Receiver and Its Performance Results}

The potential performance improvements unveiled in the previous section are inherent to the optimum detection, where the optimum receiver selects its estimate by comparing the received signal with all the possible transmitted signals. The computational load of this method is clearly too high, even considering a moderate number of subcarriers and small constellations. In this section we propose a sub-optimal receiver whose the main idea is to compare the received signal with only a set of all the possible transmitted signals. The main purpose of this receiver is achieve performance improvements closest the ones obtained by the optimum detection but, at the same time, reduce drastically the complexity associated with this type of detection. It makes uses of the fact that typically the transmitted signal differs in a few bits from the received signal and, thus, it is likely that we have the optimal sequence within the set of the tested ones without make all the possible comparisons. Our sub-optimal receiver starts its decision process by taking the hard decisions associated to the received signal. After that, it changes the first bit, modulates the resulting sequence and submits it to the same blocks whereby the received signal was submitted. Then, the Euclidean distance between the resultant signal and the received signal is evaluated. If 
this distance reduces, the bit modification is maintained, if not, the bit returns to its original value. This process is repeated for all the $\log _{2}(M) N$ bits that compose the data sequence. Moreover, it and can restart $K$ times, since the sub-optimum estimate is modified during the process. Clearly, the complexity of this algorithm is very reduced when compared to the full optimum receiver, since it will test only $\log _{2}(\mathcal{M}) N K$ sequences. An important metric to evaluate the performance of our sub-optimal receiver is the difference between its performance and the performance of the optimum receiver. However, the very high complexity of this receiver (it must analyze $2^{\log _{2}(\mathcal{M}) N}$ sequences before taking a decision) makes its simulation inviable. To obtain an approximate optimum performance, we considered to apply the behavior of the sub-optimal receiver but starting with the transmitted sequence instead of the hard-decisions sequence. Then, the selected sequence is the sequence between: the hard-decisions sequence, the transmitted sequence, or one of their modifications that has the lower Euclidean distance relatively to the received signal. Fig.3 presents the BER performance of the sub-optimal receiver described above. The considered OFDM signal has $N=64$ useful subcarriers with QPSK constelladions and an oversampling factor $M=4$. The complex data symbols were mapped under a Gray mapping rule. The nonlinear device corresponds to an ideal envelope clipping with a normalized clipping level $s_{M} / \sigma=1.0$. We considered an ideal AWGN channel and $N_{R x}$-order diversity at reception.

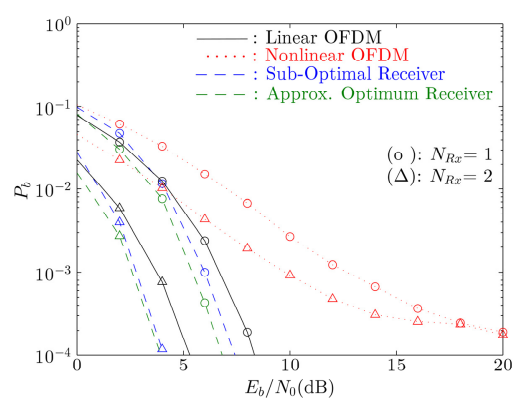

Fig. 4. Sub-Optimal receiver and approximate pptimum receiver BER in an ideal AWGN channel considering $N_{R x}$-order diversity

From Fig.4 it is clear that the sub-optimal receiver improves the performance of conventional nonlinear OFDM schemes. Without diversity and at $P_{b}=10^{-3}$, the sub-optimal receiver presents a gain of approximately $1.3 \mathrm{~dB}$ relatively to the linear OFDM. When $N_{R x}=2$ the gain is around $1.1 \mathrm{~dB}$. It is also important to note the low difference between the sub-optimal receiver performance and the approximate optimum performance. This means that even testing only a small part of all the possible transmitted signals, the performance is still close to the one potentially obtained by the optimum receiver. In Fig.4 it is shown the BER of the sub-optimal receiver considering a XTAP channel with $N_{R a y}=32$ rays of uncorrelated Rayleigh fading and $N_{R x}$-order diversity at reception. 


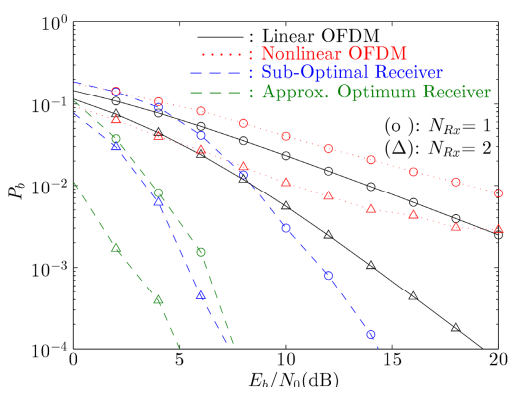

Fig. 5. Sub-Optimal receiver and Approximate Optimum receiver BER in a frequency-selective channel considering $N_{R x}$-order diversity

From Fig. 5, it is clear that independently of the diversity order, in a frequency-selective channel the gains are higher than in a ideal AWGN channel. For instance, at $P_{b}=10^{-2}$, the gain the sub-optimal receiver is around $5.4 \mathrm{~dB}$ relatively to a linear OFDM transmission and $10.3 \mathrm{~dB}$ relatively to the conventional nonlinear OFDM receiver. These higher gains can be explained due to the diversity associated to the selectivity of the channel that can be enhanced by the diversity inherent to the nonlinear distortion effects. It is also important to remark that the approximate performance of the optimum receiver is much better than the performance of our sub-optimal receiver which unveils that in a frequency-selective channel an increased complexity means better performances, however, even with a reduced complexity receiver, we have remarkable improvements relatively to the conventional nonlinear OFDM schemes.

\section{Conclusions and Further Work}

In this paper we considered the nonlinear distortion effects as useful information that can improve the performance of OFDM schemes. The adequate receiver to explore this information is the optimum receiver. However, its complexity is too high for practical applications. To overcome this problem, we present a sub-optimal receiver whose the performance is very close to the one that can be obtained by the optimum receiver but has much lower complexity. We include results with diversity and conclude that the sub-optimal detection also allows a performance improvement in these type of scenarios. The guidelines for future work can pass to theoretically derive the potential gains of the optimum (and sub-optimum) receivers under AWGN and frequency-selective channels. Another important open question is to theoretically quantify what is the impact of the filtering operation in the potential gains of the optimum-based detection.

\section{References}

1. Cimini Jr., L.: Analysis and Simulation of a Digital Mobile Channel Using Orthogonal Frequency Division Multiplexing. IEEE Trans. on Comm. 33(7), 665-675 (1985)

2. Digital Video Broadcasting (DVB); Framing structure, channel coding and modulation for digital terrestrial television, ETSI Standard: EN 300744 V1.6.1 (January 2009) 
3. 3rd Generation Partnership Project: Technical Specification Group Radio Access Network; Physical Layers Aspects for Evolved UTRA, 3GPPP TR 25.814 (2006)

4. IEEE Standard for Local and Metropolitan Area Networks - Part 16: Air Interface for Fixed Broadband Wireless Access Systems, IEEE 802.16- (October 2004)

5. Rahmatallah, Y., Mohan, S.: Peak-To-Average Power Ratio Reduction in OFDM Systems: A Survey And Taxonomy. IEEE Communications Surveys \& Tutorials (99), 1-26 (2013)

6. Dinis, R., Gusmão, A.: A Class of Nonlinear Signal Processing Schemes for Bandwidth-Efficient OFDM Transmission with Low Envelope Fluctuation. IEEE Trans. on Comm. 52(11), 2009-2018 (2004)

7. Rowe, H.: Memoryless Nonlinearities with Gaussian Input: Elementary Results. Bell System Tech. Journal, 61 (September 1982)

8. Gusmão, A., Dinis, R.: Iterative Receiver Techniques for Cancellation of Deliberate Nonlinear Distortion in OFDM-Type Transmission. In: IEEE Int. OFDM Workshop 2004, Dresden, Germany (September 2004)

9. Montezuma, P., Dinis, R., Oliveira, R.: Should we Avoid Nonlinear Effects in a Digital Transmission System? In: IEEE WCNC 2010, Sydney, Australia (April 2010)

10. Saleh, A.: Frequency-Independent and Frequency-Dependent Nonlinear Models of TWT Amplifiers. IEEE Transactions on Communications Com-29(11) (November 1981)

11. Proakis, J.G.: Digital Communications, 4th edn. McGraw-Hill (2001) 